\title{
Transport Phenomenon of Simultaneously Developing Flow and Heat Transfer in Twisted Sinusoidal Wavy Microchannel under Pulsating Inlet Flow Condition
}

\author{
Suvanjan Bhattacharyya ${ }^{1, *}$, Sampad Gobinda Das ${ }^{2}$, Himadri Chattopadhyay ${ }^{2}$, Ali Cemal Benim ${ }^{3}$, M. A. Moghimi ${ }^{4}$ \\ ${ }^{1}$ Department of Mechanical Engineering, Birla Institute of Technology and Science, Pilani, Pilani Campus, Vidya Vihar, \\ Rajasthan 333 031, India. \\ ${ }^{2}$ Department of Mechanical Engineering, Jadavpur University, Kolkata 700 32. India. \\ ${ }^{3}$ Center of Flow Simulation (CFS), Department of Mechanical and Process Engineering, Dusseldorf University of \\ Applied Sciences, D-40476 Dusseldorf, Germany. \\ ${ }^{4}$ Department of Design \& Engineering, Staffordshire University, Stoke-on-Trent, UK, ST4 2DE.
}

\begin{abstract}
The transport phenomena in microchannel are significant in designing MEMS devices. The current study investigates numerically the simultaneously developing unsteady laminar flow and heat transfer inside a twisted sinusoidal wavy microchannel. At the inlet sinusoidal varying velocity component is applied. Varying pulsating amplitude and frequency represented by the Strouhal number was studied for Reynolds numbers ranging from 1 to 100 . The governing equations are solved with a finite volume based numerical method. In comparison with steady flow, it was found that imposed sinusoidal velocity at the inlet can provide improved heat transfer performance at different amplitudes and frequencies while keeping the pressure drop within acceptable limits.
\end{abstract}

\section{Introduction}

In the modern era of high speed computing and intensive use of integrated circuits, the thermal management of devices has become a key area where improvements can be brought. Better thermal management option for a system can increase its durability and efficiency, as well as showing a way of possible lesser power consumption which has become a point of attraction in these days. With day-by-day increased use and miniaturisation of electronic devices, use of micro and mini devices, their thermal management has become point of concern. Researchers observed that, decreasing channel dimensions to microscale, can lead to increase in heat transfer rates as well as improving heat-sinking capability. Microchannel heat sinks (MCHS) represent an innovative cooling technology for the dismissal of large heat through a small area. It provides very high surface area to volume ratio, which enhances heat transfer rate.

Extensive researches on micro-channel heat exchangers (MCHE) has been done till date. In their pioneering work, Tuckerman \& Pease [1] observed thermal resistance of $0.09^{\circ} \mathrm{C} / \mathrm{W}$. They worked on rectangular micro-channel having width of $50 \mu \mathrm{m}$ and $302 \mu \mathrm{m}$ depth. Water was used as the cooling medium and a constant heat flux of $790 \mathrm{~W} / \mathrm{cm} 2$ was applied.
Kandilkar et al. [2] reported in details about heat exchange characteristics and the flow behavior for micro-channels and mini-channels. A comparison of different cooling technologies was presented by Kheirabadi et al. [3]. The work examined liquid cooling; spray cooling, air-cooling, pool boiling, heat pipes and jet impingement. It was predicted that by the year 2020, liquid cooling would be the norm for high performance computing, whereas air-cooling will be viable for general purpose computing.

Although heat transfer enhancement by means of flow pulsation for turbulent forced convection was a subject of investigation since many years [5], the effect of pulsatile flow on microchannel heat transfer received rather recently attention. Nandi and Chattopadhyay [4] found enhanced performance in microchannel heat transfer as a result of introduction of pulsation. They also presented a detail study of effect frequency and amplitude on thermal performance of MCHE. Leng et al. [6] numerically experimented and proposed design improvement in micro-channel heat sink having double layers. Upon investigating simultaneously developing flow in a microchannel using pulsating flow at inlet.

The friction factor, convective heat transfer coefficient and the effects of inner wall surface roughness for laminar and turbulent flow in micro tubes 
were studied by Choi et al [7]. The experimental results were significantly different from the correlations in the conventional theories. Kazmierezak et al. [8] experimented for single-phase forced convention in deep rectangular micro channels. Two configurations were tested, a single channel system and multiple channel system. In the case of the multiple channel system the channels were $251 \mu \mathrm{m}$ wide and the channel walls were $119 \mu \mathrm{m}$ thick. In both systems the channels were approximately $1000 \mu \mathrm{m}$ deep and defined a projected area of $2.5 \mathrm{~cm} \times 2.5 \mathrm{~cm}$. Deionized water was used as the working fluid, Reynolds number ranged from 173 to 12900. The experimentally obtained local Nusselt number agrees reasonably well with classical developing channel flow theory. An experimental and theoretical investigation on single-phase heat transfer in micro channels was done by Hetsroni et al [9]. They discussed several aspects of flow in micro-channels as pressure drop, transition from laminar to turbulent etc. They considered the problem of heat transfer corresponding to small Knudsen number. They analysed the data of heat transfer in micro-channels with hydraulic diameters ranging from $60 \mu \mathrm{m}$ to $200 \mu \mathrm{m}$. They also discussed the effects of geometry, axial heat flux due to thermal conduction through the working fluid and channel walls as well as the energy dissipation. They found that the effect of energy dissipation on heat transfer in microchannels is negligible under typical flow conditions. Judy et al. [10] performed experiments on both round and square micro channels with hydraulic diameters ranging from 15 to $150 \mu \mathrm{m}$. They tested distilled water, methanol, and isopropanol over a Reynolds number range of 8 to 2300. Their results showed no distinguishable deviation from laminar flow theory.

Flow visualization and pressure drop studies on micro-channels, conducted by Liu and Garimella [11], with hydraulic diameters ranging from 244 to $974 \mu \mathrm{m}$ over a Reynolds number range of 230 to 6500 . They were able to measure the onset of turbulence through their flow visualization, and compared their pressure drop measurements with numerical calculations. Their results showed that both conventional turbulent transition and pressure drop correlations are valid on the micro scale. Upon experimenting micro channels of 349 $\mu \mathrm{m}$ hydraulic diameter, $\mathrm{Qu}$ and Mudawar [12] found that the friction factor data agreed well with classical theory. Gogineni et al [13] investigated for laminar convective heat transfer co-efficient in a rectangular micro-channel under constant wall heat flux. They worked on Reynolds Number ranging between $100-400$. They found high heat transfer intensity near the channel inlet while it is decreasing towards the downstream. A 3D rectangular channel with vortex promoters was investigated by Icoz and Jaluria [14]. The vortex promoters had circular, square, and hexagonal shapes, and were located ahead of two tandem-heating sources. Different blockage ratios were considered. They reported that the hexagonal shape is best to remove heat from the first heat source and the circular one is optimal when heat removal from the second source is the main aim. The square vortex promoter gave a reasonable combination of heat transfer and pressure drop. Meis et al. [16] studied the heat transfer enhancement in micro-channels using vortex promoters. Here a laminar 2D flow of water was considered with vortex promoters of circular and rectangular shapes. Different designs were studied and a useful design was chosen which both thermal efficient and not expensive in terms of pumping power based on the plots of thermal efficiency and pressure drop.

Mohammed et al. [15] studied heat transfer enhancement and friction factor in a wavy micro-channel and reported that with increase in wave amplitude of the channel, the friction factor also increases. Goldstein et al. [17] found that separation and reattachment significantly affects the heat transfer augmentation while experimenting in a corrugated wall channel. Rush et al. [18] investigated for heat transfer enhancement through wavy channels and found higher enhancement near the exit of the channel. They also reported that, with increase in Reynolds number, the highly enhanced zone shifted towards the channel entrance. Upon investigating on sinusoidal corrugated plate channels, Metwally et al. [19] found that the waviness of the channel enhances the generation of transverse vortices and it increases with increase in the Reynolds number. Experimental and numerical investigations on serpentine micro-channel were done by Al-Neame et al. [20]. They found an increase in heat transfer about $35 \%$ and reduction of total thermal resistance about 19\%. Patankar et al. [21] showed in their numerical study in periodically varrying cross-section ducts, that there are huge recirculation zones due to the varying cross sections. Thermal performance of converging-diverging micro-channels was studied by Ghaedamini et al. [22]. They found better thermal performance with increase in waviness. Xie et al. [23] executed heat transfer investigations in transverse wavy micro-channel. They found enhanced heat transfer rates coupled with reduced pressure drop. Masliyah et al. [24] studied heat transfer enhancement in square twisted tube. They found that the twisted nature of the channel enhances swirl generation and better mixing of the fluid, thus enhancing heat transfer. Bhattacharyya et al. [25-35] studied the effects of inserts on the overall heat transfer. The analysis was carried out in the all three flow region, with the results of thermal performance greatly supporting the use of inserts as a means of augmenting heat transfer.

From the above literature survey, it is evident that the prior works mainly focused on geometrical modification of the flow passage and moderate thermal performance comes at the cost of a high pressure drop. Studies on the use of flow pulsation at inlet (active technique) are very rare. Based on the above findings, the present study aims to work on both of them (i.e. twisted flow passage to pertain out of plane mixing and flow pulsation at inlet). Three-dimensional micro-channels under pulsating flow at inlet are investigated in the present study. The main aim was to enhance heat transfer rate by increasing recirculation and mixing of the flowing fluid. 


\section{Geometry}

Fig. 1 shows the schematic of the sinusoidal wavy microchannel. The height of the channel follows a path as per the following function

$$
y=A_{w} \sin \left(\frac{2 \pi}{\lambda} x\right)
$$

The channel cross section was a square of $0.50 \mathrm{~mm}$ breadth. The channel was made $25 \mathrm{~cm}$ long and the wave amplitude $\left(\mathrm{A}_{\mathrm{w}}\right)$ of the channel was taken as $1 \mathrm{~mm}$. The channel was made taking a pitch (S) of $5 \mathrm{~cm}$ and wavelength $(\lambda)$ as $2.5 \mathrm{~cm}$.

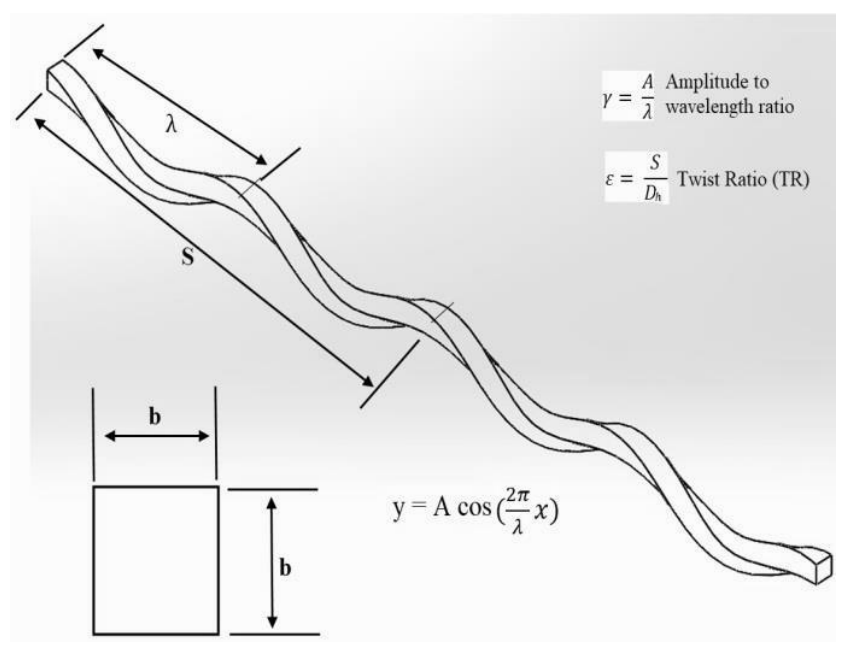

Fig. 1. Computation domain.

\section{Mathematical and Numerical Description}

The following assumptions were made for the mathematical description of the flow: (1) flow is unsteady, (2) flow is laminar, (3) flow is incompressible, (4) radiation heat transfer is negligible [36], (5) viscous dissipation is negligible, (6) body forces are negligible, and (7) material properties are constant. Based on these assumptions, the following governing equations describe the flow.

Continuity Equation

$$
\frac{\partial u_{i}}{\partial t}+\nabla \cdot(\rho u)=0
$$

Momentum Equation

$$
\frac{\partial \mathrm{u}_{\mathrm{i}}}{\partial \mathrm{t}}+\frac{\partial\left(\mathrm{u}_{\mathrm{i}} \mathrm{u}_{\mathrm{j}}\right)}{\partial \mathrm{x}_{\mathrm{i}}}=-\frac{\partial \mathrm{p}}{\partial \mathrm{x}_{\mathrm{i}}}+\frac{1}{\mathrm{R}_{\mathrm{e}}} \nabla^{2} \mathrm{u}_{\mathrm{j}}
$$

Energy Equation

$$
\frac{\partial T}{\partial t}+u_{i} \frac{\partial T}{\partial x_{i}}=\frac{1}{R_{e} P r} \nabla^{2} T
$$

p: static pressure, Re: Reynolds number, Pr: Prandtl number, $\mathrm{t}$ : time, $\mathrm{T}$ : static temperature, $\mathrm{u}_{\mathrm{i}}$ : the velocity vector, $\mathrm{x}_{\mathrm{i}}$ : Cartesian coordinates, $\rho$ : density)

On the walls, no slip boundary conditions prevail for the momentum equations, while assuming an isothermal boundary for the energy equation $\left(\mathrm{T}_{\mathrm{w}}=330 \mathrm{~K}\right)$. At the inlet, the velocity is assumed to be spatially uniform, but variably in time. A sinusoidal velocity fluctuation was added to the uniform velocity profile to obtain a sinusoidally variable (time dependent) flow at the inlet. The inlet velocity can be represented by the following equation:

$$
u_{i n}=u_{m}\{1+A \sin (2 \pi f t)\}
$$

Where, $\mathrm{u}_{\text {in }}$ is the mean velocity and $\mathrm{A}$ is the amplitude. The frequency of the inlet pulsation comes from the dimensionless Strouhal number $(\mathrm{St})$ which is defined as $\mathrm{St}=\mathrm{f} . \mathrm{D}_{\mathrm{h}} / \mathrm{u}_{\mathrm{m}}$.

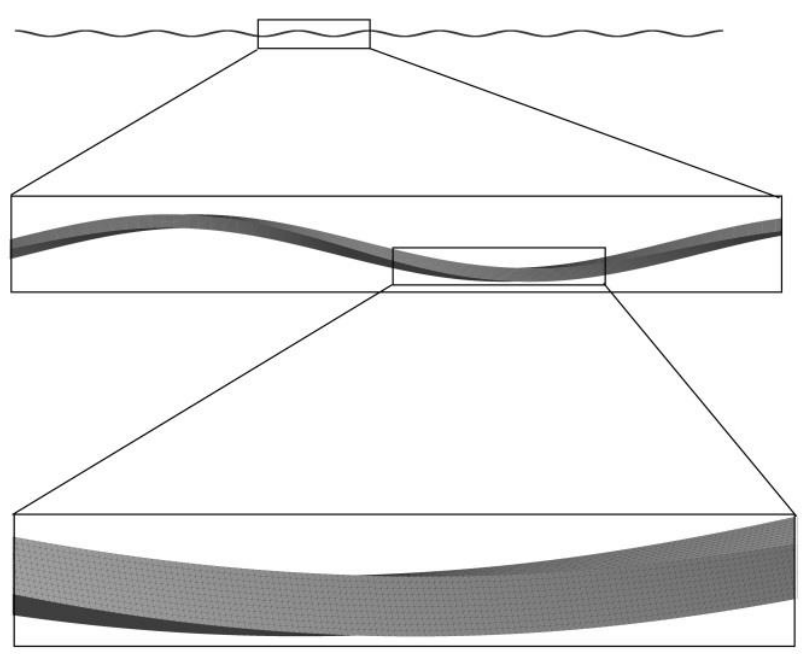

Fig. 2. Meshing of computational domain.

The commercial Package ANSYS Fluent 19.2 was used for the investigation purpose, which is based on the finite volume based method. To examine the effect of Reynolds number and amplitude of the inlet pulsation, a range of Reynolds numbers $(1-100)$ and amplitudes $(0.10-1.00)$ were investigated. The expanded form of the governing equation and solution procedure may be found in Nandi and Chattopadhyay [5].

An unstructured grid with non-uniform structure density distribution was created. After performing a grid independence study, a grid with 758693 nodes was identified to deliver sufficient grid independent results and, thus, chosen for computation. A close view of the meshing is shown in Fig. 2. 
The computational model was verified by computing developing flow in the tested microchannel. In the current study, studies were performed within the range of $1 \leq \mathrm{St} \leq 10$ and $0.1<\mathrm{A}<1$ corresponding to typical microchannel study. The observed data plot in Fig. 3 showing the instantaneous axial velocity in the middle of the channel confirms that that the flow field is fully periodic, following the periodic pulsation at the inlet.

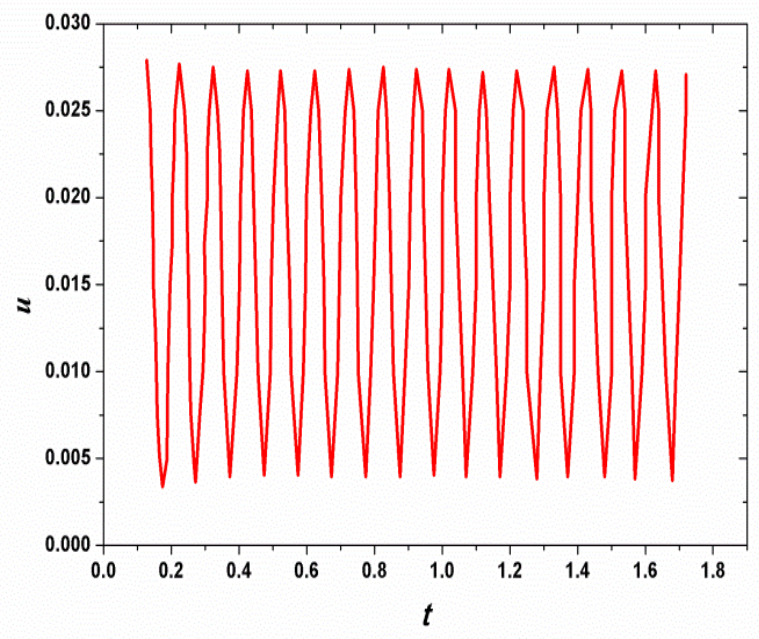

Fig. 3. Periodical local axial velocity

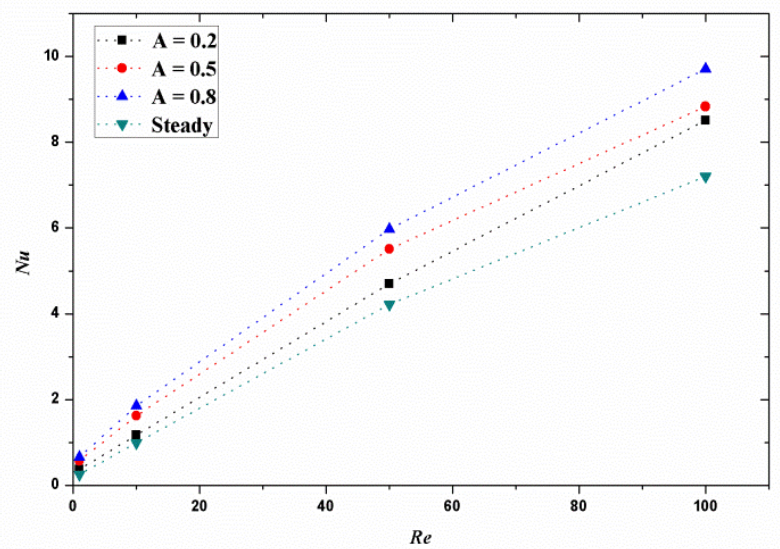

Fig. 4. Comparison of time average $\mathrm{Nu}$ with $\mathrm{Re}$ for different amplitudes.

\section{Results and Discussion}

Computations were carried out in the range $1 \leq \mathrm{St} \leq 10$ and $0.10 \leq \mathrm{A} \leq 1$. Time averaged data (e.g. time averaged $\mathrm{Nu}$ and $f$ values in the developing region) were used for the calculations.

It is evident from Fig. 4 that at very low $R e$ and low amplitude (A) of the inlet pulsation, the average Nusselt number values are very close to that of a steady case. This is caused by the weak swirl generation at low Reynolds number $(R e)$ as the viscous force predominates in that case over the effect of the channel geometry.
With increase is pulsation amplitude (A) and Re, augmentation of heat transfer and hence greater values of $\mathrm{Nu}$ are found. This is due to better mixing of fluid caused by the flow pulsation and recirculation generated by the twisted and wavy nature of the channel geometry. The steady one is also showing minimum heat transfer. The current work is compared with the established work of Nandi and Chattopadhyay [5] in Fig. 5 and the current geometry shows better results.

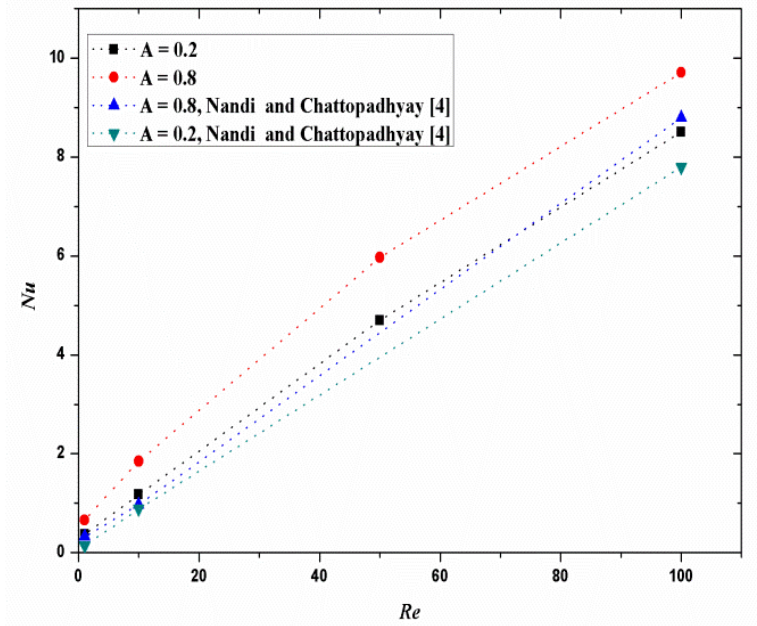

Fig. 5. Comparison of time average $\mathrm{Nu}$ with $\mathrm{Re}$ with previous study

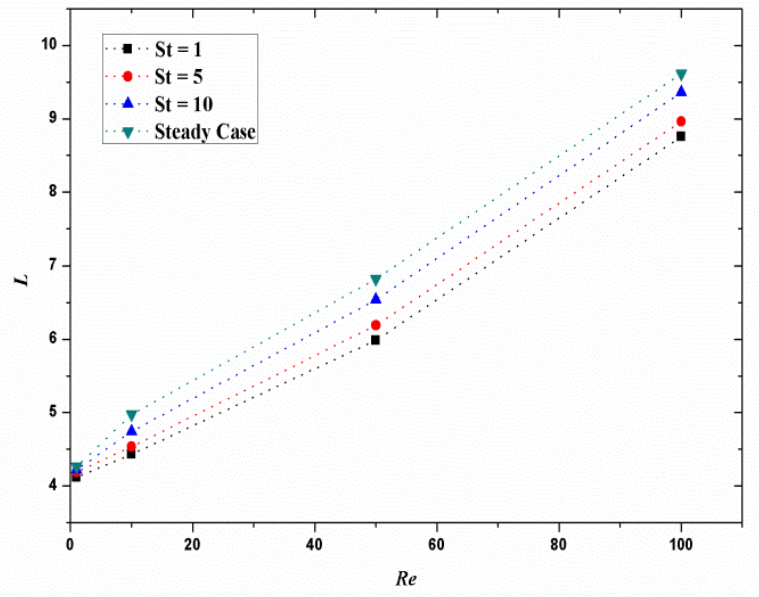

Fig. 6 Comparison of thermal development length with varying Re for different $\mathrm{St}$.

Thermal development length (L) is an important parameter which was examined for a range of $R e$ and Strouhal numbers $(\mathrm{St})$ in the present study. Fig. 6 shows the thermal development length as a function of Re for different Strouhal numbers with increase in St, it shows shorter development length as compared to the steady case. This is the result of inlet flow pulsation and better mixing of near wall fluid with the core fluid.

Fig. 7 shows variation of the friction factor as a function of the pulsation amplitude for different frequency and its 
comparison with previous well-established works and steady case. It is evident from the figure that the current geometry is providing better thermal result with minimal increased pressure drop. Also, one can see that the steady case is showing higher friction factor $f$ in comparison with pulsating flow situations.

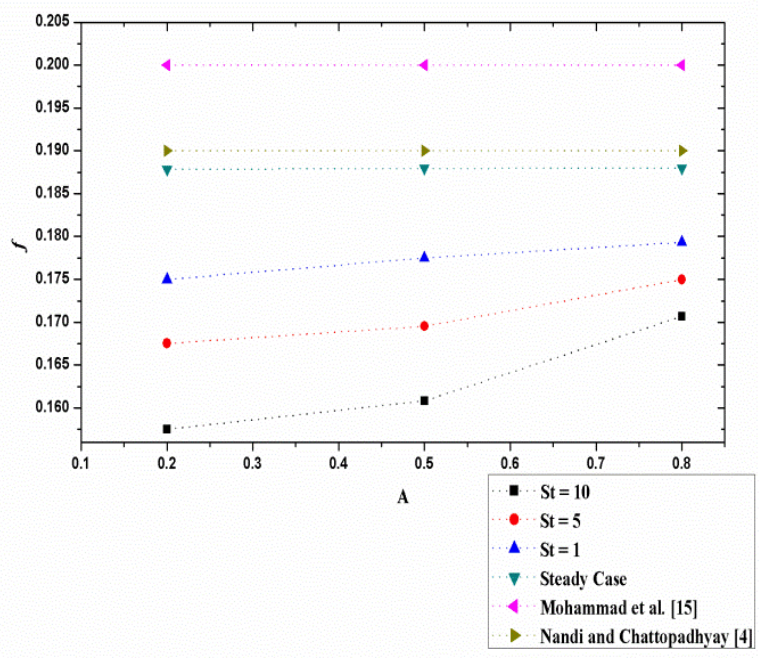

Fig. 7. Variation of $\mathrm{f}$ with $\mathrm{A}$ for different $\mathrm{St}$ at $\mathrm{Re}=100$.

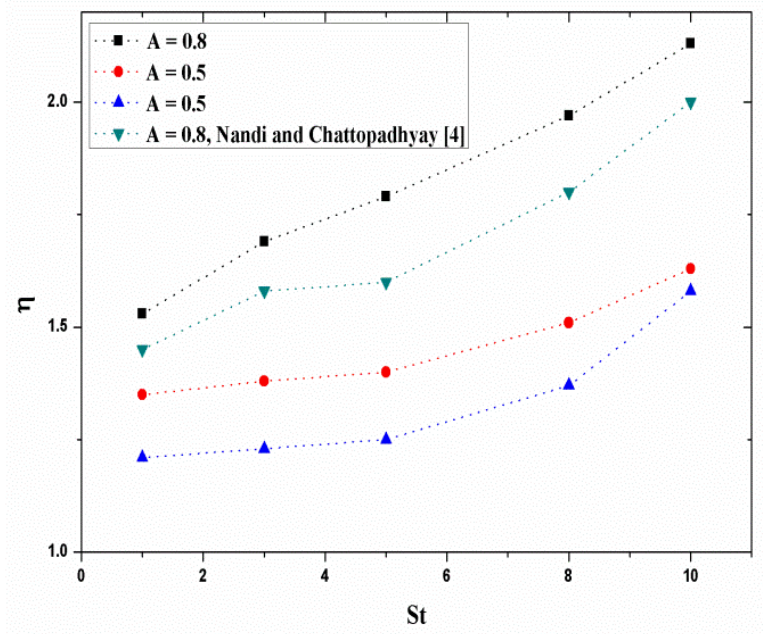

Fig. 8. Comparison of enhancement ratio with St for different $A$ at $\operatorname{Re}=100$.

Performance enhancement ratio $(\eta)$ is defined as $\eta=$ $\left(N u_{\text {avg }} / N u_{s}\right)$. Here, $\mathrm{Nu}_{\mathrm{avg}}$ is the time averaged value of local $N u$ at a particular position and $N u_{s}$ is the value for steady case at same position. It is the measure of thermal performance of the proposed geometry. $\eta$ as a function of St at different A and constant Re is shown in Fig. 8. The results were also compared with the previous work of Nandi and Chattopadhyay [5]. Figure shows that at Re $=100, \mathrm{St}=10$ provides the maximum efficiency. This is due to the inlet flow pulsation which is overlaid with the core fluid flow of the micro-channel, causing the flow being unsteady enough to provide proper mixing of fluid.

\section{Conclusion}

Effects of pulsatile flow inside sinusoidal wavy microchannel was investigated by solving the solution of transient 3D Navier - Stokes equations numerically. Sinusoidal fluctuating velocity components were added to the mean flow at inlet. The channel wall was kept at constant temperature. Enhanced thermal performance was found as a result coupled with minimal pressure penalty even at low Re. The effect of flow pulsation on thermal development lengths was also examined.

\section{References}

1. D. B. Tuckerman, R.F.W. Pease, Highperformance heat sinking for VLSI, EEE Electron Device Letters, 2(5), 126-129 (1981).

2. S.G., Kandlikar, M.R., King, Heat transfer and fluid flow in mini-channels and micro-channels, S.G.K.G.L.C.R. King (Ed.) (2006).

3. A.C. Kheirabadi, D. Groulx, , Cooling of server electronics: a design review of existing technology, Appl. Therm. Engg., 105, 622-638 (2016).

4. T.K. Nandi, H. Chattopadhyay, Numerical investigation of simultaneously developing flow in wavy microchannel under pulsating inlet flow condition, Int. Commun. Heat Mass Transf., 47, 27-31 (2013).

5. A.C. Benim, M.. Cagan, D. Gunes, Computational analysis of transient heat transfer in turbulent pipe flow, Int Jour of Therm Sc, 43(8), 725-732 (2004).

6. C. Leng, X.D. Wang, T.H. Wang, An improved design of double-layered micro-channel heat sink with truncated top channels, Appl. Therm. Engg., 79, 54-62 (2015).

7. S.B. Choi R.R. Baron, R.O. Warrington, Fluid flow and heat transfer in micro-tubes, ASME, DSC 40, 89-93 (1991).

8. T. M. Harms, M. J. Kazmierczak, F. M. Gerner Developing convective heat transfer in deep rectangular micro channels, Int Jour of Heat and Fluid Flow, 149-157 (1999).

9. G. Hetsroni, A. Mosyak, E. Pogrebnyak, L. P. Yarin, Heat transfer in microchannel comparison of experiments with theory and numerical results, Int. J. Heat Mass Trans, 48, 5580-5601 (2005).

10. J. Judy, D. Maynes, B.W. Webb, Characterization of frictional pressure drop for liquid flows through microc-hannels, Int Jour of Heat and Mass Trans, 45(17), 3477-3489 (2002).

11. D. Liu, S.V. Garimella, Investigation of liquid flow in micro-channels, AIAA Jour of Thermophysics and Heat Trans, 18, 65-72 (2004).

12. W. Qu, I. Mudawar, Experimental and numerical study of pressure drop and heat transfer in a single-phase microchannel heat 
sink, Int Jour of Heat and Mass Trans, 45, 2549-2565 (2002).

13. A. Gogineni, CFD Modelling Of Rectangular Microchannel With Increase In Heat Flux And Effect On Nusselt Number, Proceedings of the 6th Annual GRASP Symposium, Wichita State University (2010).

14. T. Icoz, Y. Jaluria, Design optimisation of size and geometry of vortex promoter in a twodimensional channel, J. Heat Trans, 128, 10811092 (2006).

15. H. A. Mohammed, P. Gunnasegaran, N.H. Shuais, Numerical simulation of heat transfer enhancement in wavy microchannel heat sink, Int. Commun. Heat Mass Transf., 38, 63-68 (2011).

16. M. Meis, F. Varas, A Velázquez, J. M. Vega Heat transfer enhancement in micro-channels caused by vortex promoters, Int Jour of Heat and Mass trans, 53, 29-40 (2009).

17. L., Goldstein, E. Sparrow, Heat/mass transfer characteristics for flow in a corrugated wall channel, ASME Trans J Heat Tran, 99, 187-195 (1977).

18. T. A. Rush, T.A. Newell, A.M. Jacobi, An experimental study of flow and heat transfer in sinusoidal wavy passages, Int $\mathrm{J}$ Heat Mass Tran, 42 (9), 1541-1553 (1999).

19. H. M. Metwally, M.R. Manglik, Enhanced heat transfer due to curvature-induced lateral vortices in laminar flows in sinusoidal corrugated-plate channels, Int J Heat Mass Tran, 47 (10), 2283-2292 (2004).

20. A.F. Al-Neama, N. Kapur, J. Summers, H. M. Thompson, An experimental and numerical investigation of the use of liquid flow in serpentine microchannels for microelectronics cooling, Appl. Therm Eng., 116, 709-723 (2017).

21. S. Patankar, C. Liu, E. Sparrow, Fully developed flow and heat transfer in ducts having stream wise-periodic variations of crosssectional area, ASME J Heat Tran, 99 (2), 180186 (1977).

22. H. Ghaedamini, P.S. Lee, C. J. Teo, Developing forced convection in converging-diverging microchannels, Int J Heat Mass Tran, 65, 491499 (2013).

23. G. Xie, J. Liu, Y. Liu, B. Sunden, W. Zhang, Comparative study of thermal performance of longitudinal and transversal-wavy microchannel heat sinks for electronic cooling, J Electron Package, 135 (2), 21008 (2013).

24. J. Masliyah, K. Nandakumar, Steady laminar flow through twisted pipes: fluid flow in square tubes, J Heat Tran, 103 (4),785-790 (1981).

25. S. Bhattacharyya, H. Chattopadhyay, A. Swami, Md. Kamal Uddin, Convective Heat Transfer Enhancement and Entropy Generation of Laminar Flow of Water Through a Wavy
Channel, Int Jour of heat and Tech, Vol. 34 (4), 727-733 (2016).

26. S. Bhattacharyya, H. Chattopadhyay, A. C. Benim, Numerical Investigation on Heat Transfer in a Circular Tube with Inclined Ribs, Progress in Comp Fl Dyn, An International Journal, Vol. 17, no. 6, 390396. (2017).

27. S. Bhattacharyya, H. Chattopadhyay, A. Guin, A. C. Benim, Investigation of inclined turbulators for heat transfer enhancement in a solar air heater, Heat Tran Engg, Vol. 40 no. 17-18, 1451-1460 (2019).

28. S. Bhattacharyya, A. C. Benim, Himadri Chattopadhyay, A. Banerjee, Experimental Investigation of the Heat Transfer Performance of a Corrugated Tube with Spring tape inserts. Exp. Heat Transfer Vol. 32, no. 5, 411425. (2019).

29. S. Bhattacharyya, Experimental study on effect of heat transfer enhancement of heat exchanger tube inserted with short length spring tapes, Iran J Sci Technol Trans Mech Engg, Online Published. (2018).

30. S. Bhattacharyya, A. C. Benim, H. Chattopadhyay, A. Banerjee, Experimental and numerical analysis of forced convection in a twisted tube, Jour of Therm Sc ( Accepted) (2019).

31. R. Biswas, M. C. Das, S. Bhattacharyya, A. S. Kuar, S. Mitra, Selection of Nd:YAG laser beam micro-drilling parameters using multicriteria decision making methods, Optics \& Laser Tech, 119, 105596. (2019).

32. S. Bhattacharyya, A. I. Bashir, K. Dey, R. Sarkar, Effect of Novel Short Length Wavy Tape Turbulators on Fluid Flow and Heat Transfer: Experimental Study, Exp Heat Transf Online Published, (2019).

33. S. Bhattacharyya, The effects of short length and full length swirl generators on heat transfer and flow fields in a solar air heater tube, Jour of Therm Analysis and Calorimetry (Online Published), (2019).

34. S. Bhattacharyya, H. Chattopadhyay, S. K. Saha, Numerical Study on heat transfer enhancement of laminar flow through a circular tube with artificial rib roughness, Journal of Ref Air cond Heating and vent, 1 (3), 14-19 (2014).

35. S. Bhattacharyya, S. Das, A Sarkar, A Guin, A.Mullick, Numerical simulation of flow and heat transfer around hexagonal cylinder, Int Jour of Heat and Tech, 35 (2), 360-363 2016).

36. A. C. Benim, A finite element solution of radiative heat transfer in participating media utilizing the moment method, Comp Methods in App Mech and Engg, 67(1), 1-14 (1988). 